\title{
Positivity of Thom polynomials II: the Lagrange singularities
}

by

Małgorzata Mikosz, Piotr Pragacz and Andrzej Weber (Warszawa)

\begin{abstract}
We study Thom polynomials associated with Lagrange singularities. We expand them in the basis of $\widetilde{Q}$-functions. This basis plays a key role in the Schubert calculus of isotropic Grassmannians. We prove that the $\widetilde{Q}$-function expansions of the Thom polynomials of Lagrange singularities always have nonnegative coefficients. This is an analog of a result on the Thom polynomials of mapping singularities and Schur $S$-functions, established formerly by the last two authors.
\end{abstract}

1. Introduction. In the present paper, we study the Thom polynomials of Lagrange singularities. This is a continuation of [16], where the case of singularities of maps was investigated. We look at the positivity properties of Thom polynomials. Such positivity properties are nowadays widely investigated in algebraic geometry (see the monograph of Lazarsfeld [13]).

Let $L$ be a Lagrangian submanifold in the linear symplectic space

$$
V=W \oplus W^{*},
$$

equipped with the standard symplectic form. Classically, in real symplectic geometry, the Maslov class ([1]) is represented by the cycle

$$
\Sigma=\left\{x \in L: \operatorname{dim}\left(T_{x} L \cap W^{*}\right)>0\right\} .
$$

This cycle is the locus of singularities of the projection $L \rightarrow W$. It defines an integral cohomology class whose reduction modulo 2 is equal to $w_{1}\left(T^{*} L\right)$, the first Stiefel-Whitney class. In complex symplectic geometry the same construction applied for a Lagrangian submanifold $L$ contained in a symplectic manifold fibering over a base $B$ with Lagrangian fibers leads to the cohomology class which is equal to

$$
c_{1}\left(T^{*} L-T^{*} B\right) \text {. }
$$

2000 Mathematics Subject Classification: 05E05, 14C17, 14N15, 55R40, 57R45.

Key words and phrases: Lagrange singularities, Thom polynomials, $\widetilde{Q}$-functions, jets, numerical positivity.

Research of M. Mikosz and A. Weber supported by the KBN grant NN201 387034 . 
A generalization of the Maslov class is provided by Thom polynomials associated with higher order types of singularities. These types are defined by imposing conditions on higher order jets of $L$ (see Definition 3 ).

For real singularities, the associated cohomology classes were studied by Arnold and Fuks (see, e.g., [5]), Vassiliev [17], Audin [3], and others. The complex case was studied by Kazarian [11] who also computed a substantial number of examples. The Thom polynomials in this case can always be written as polynomials in the Chern classes of $T^{*} L \rightarrow T^{*} B$ (see Remark 13).

Given a Thom polynomial, one can expand it in different bases. The Thom polynomial of a singularity of a map, in general, is not a positive combination of monomials in Chern classes. As shown in [16], such a Thom polynomial is always a positive $\mathbb{Z}$-linear combination of Schur $S$-functions.

The Thom polynomial of a Lagrange singularity is, in general, neither a positive combination of monomials in Chern classes nor a positive combination of Schur $S$-functions.

In the present paper, we use the $\widetilde{Q}$-functions of [15] and show that the Thom polynomial of a Lagrange singularity, expanded in the basis of $\widetilde{Q}$ functions, has nonnegative coefficients.

Here is a brief outline of the content of the paper.

In Section 2, we recall the definitions and properties of Lagrange singularities. We introduce the space of jets of Lagrange submanifolds and define the notion of a "Lagrange singularity type".

In Section 3, we recall from [15] the algebraic properties of $\widetilde{Q}$-functions. We also recall from [14] their cohomological interpretation in terms of Schubert classes for the Lagrangian Grassmannian.

In Section 4, we attach the "Thom polynomial" to a Lagrange singularity type. Then we state and prove our main result, Theorem 9, asserting that the Thom polynomial of a Lagrange singularity type is a nonnegative $\mathbb{Z}$-linear combination of $\widetilde{Q}$-functions.

The proof of the theorem is quite different from the one in [16]. There are two reasons for that. First, the $\widetilde{Q}$-functions do not admit-up to now-a characterization similar to that of Schur $S$-functions from [8]. Second, the Lagrangian case is "more rigid" than the one of singularities of maps, and does not admit a functorial interpretation like that in [16]. The proof in the present paper relies on the computation, in Lemma 12, of some normal bundle in the space of jets of Lagrangian submanifolds. This lemma is based on a result about actions of linear transformations on jets of functions (see Proposition 11) which seems to be of independent interest. In the key step, the proof uses deformation to the normal cone.

We finish Section 4 with some discussion of Thom polynomials of Legendre singularities. 
In the last section, we list the $\widetilde{Q}$-function expansions of Thom polynomials up to codimension 6 .

2. Jets of Lagrangian submanifolds. Let us fix a positive integer $n$. Suppose that $W$ is a complex linear space, where $\operatorname{dim} W=n$. Let

$$
V=W \oplus W^{*}
$$

be a linear symplectic space, equipped with the standard symplectic form $\langle-,-\rangle$, defined by

$$
\left\langle\left(w_{1}, f_{1}\right),\left(w_{2}, f_{2}\right)\right\rangle=f_{1}\left(w_{2}\right)-f_{2}\left(w_{1}\right)
$$

for $w_{i} \in W$ and $f_{i} \in W^{*}, i=1,2$. We shall view $V$ as a symplectic manifold. If we write $q=\left(q_{1}, \ldots, q_{n}\right)$ for the coordinates of $W$ and $p=\left(p_{1}, \ldots, p_{n}\right)$ for the dual coordinates of $W^{*}$, the symplectic form on $V$ is $\sum_{i=1}^{n} d p_{i} \wedge d q_{i}$.

Denote by $\varrho: V \rightarrow W$ the projection.

Any germ $L$ of a Lagrangian submanifold of $V$ with the nonsingular projection $\varrho_{\mid L}$ is the graph of a 1 -form $\alpha: W \rightarrow W^{*}$. The condition that $L$ is Lagrangian is equivalent to $d \alpha=0$. Since we deal with germs (in fact, with their jets), we can write $\alpha=d F$ for some function $F: W \rightarrow \mathbb{C}$.

In the present paper, we shall investigate (the germs of) singular Lagrangian submanifolds $L$, that is, $L$ itself is smooth but the projection $\varrho_{\mid L}$ is singular.

Here is the simplest example. Suppose that $\operatorname{dim} W=1$, and set

$$
L=\left\{(q, p) \in W \oplus W^{*}: q=p^{2}\right\} .
$$

(In the classification of Lagrange singularities, this corresponds to the singularity of type $A_{2}$.)

To classify the (germs of) Lagrangian submanifolds, one introduces a suitable notion of a generating family of a Lagrangian germ (for motivation, see [2, Example 5 in $§ 18.3]$, and for a precise definition, see [2, §19.1]). (These generating families are usually versal deformations of suitable functions $f: \mathbb{C}^{\bullet} \rightarrow \mathbb{C}$ (loc. cit.).) Then one introduces, in $[2, \S 19.4]$, the notions of $R$-equivalence and (stable) $R^{+}$-equivalence of two generating families of Lagrangian germs. The crucial result (see [2, Theorem in §19.4, p. 304]) asserts that the germs of two Lagrangian submanifolds are Lagrangian equivalent if and only if the corresponding generating families are stably $R^{+}$-equivalent. We illustrate these issues by the following example.

EXAMPLE 1. The Lagrangian submanifold corresponding to the singularity $A_{3}$, i.e. $f(x)=x^{4}$, is obtained in the following way. The generating family

$$
F(x, q)=x^{4}+q_{1} x^{2}+q_{2} x
$$


is a universal deformation of $f(x)=F(x, 0)=x^{4}$ (with additional condition $F(0, q)=0)$. This generating family defines the Lagrangian submanifold

$$
L=\left\{(q, p) \in W \oplus W^{*}: \exists x \in \mathbb{C}, \frac{\partial F(x, q)}{\partial x}=0, \frac{\partial F(x, q)}{\partial q}=p\right\},
$$

that is,

$$
4 x^{3}+2 q_{1} x+q_{2}=0, \quad x^{2}=p_{1}, \quad x=p_{2} .
$$

We thus obtain

$$
L=\left\{(q, p) \in W \oplus W^{*}: p_{1}=p_{2}^{2}, q_{2}=-\left(4 p_{2}^{3}+2 q_{1} p_{2}\right)\right\} .
$$

We will study the space of germs of Lagrangian submanifolds $L \subset V$ passing through 0 . This space has infinite dimension, which is inconvenient from the point of view of algebraic geometry. Therefore we fix once for all a nonnegative integer $k$ and we identify two germs if the degree of their tangency at 0 is greater than $k$. This way we obtain the space of $k$-jets of Lagrangian manifolds, denoted by $\mathcal{L}(V)$. This space is homogeneous. Every germ of a Lagrangian submanifold can be obtained from the "distinguished" Lagrangian submanifold $W$ by application of a germ symplectomorphism preserving 0 . We have the following description:

$$
\mathcal{L}(V)=\operatorname{Aut}(V) / P
$$

where $\operatorname{Aut}(V)$ is the group of $k$-jet symplectomorphisms preserving $0 \in V$, and $P$ is the stabilizer of $W$.

Denote now by $L G(V)$ the Lagrangian Grassmannian parametrizing all linear Lagrangian subspaces of $V$. This manifold is embedded in $\mathcal{L}(V)$ in a natural way.

On the other hand, we have the Gauss map $\pi: \mathcal{L}(V) \rightarrow L G(V)$, which is a retraction to $L G(V)$, defined for a Lagrangian submanifold $L$ by

$$
\pi(L)=T_{0}(L),
$$

the tangent space to $L$ at $0 \in L$.

LEMMA 2. The fiber of the projection $\pi$ is the affine space isomorphic to $\bigoplus_{i=3}^{k+1} \operatorname{Sym}^{i}\left(W^{*}\right)\left({ }^{1}\right)$.

Proof. The fiber $\mathcal{L}(V)_{W}=\pi^{-1}(W)$ consists of those (jets of) Lagrangian submanifolds which have the tangent space equal to $W$. Every Lagrangian submanifold $L$ with nondegenerate projection onto $W$ is the graph of the differential of a function $F: W \rightarrow \mathbb{C}$ (note that $d F$ acts from $W$ to $W^{*}$ ). The

$\left({ }^{1}\right)$ We point out that $\pi: \mathcal{L}(V) \rightarrow L G(V)$ is not a vector bundle. This projection is of the form

$$
\mathcal{L}(V)=S p(V) \times_{P^{\prime}} \mathcal{L}(V)_{W} \rightarrow S p(V) / P^{\prime}=L G(V),
$$

where $P^{\prime}=P \cap S p(V)$ is the group of linear symplectomorphisms stabilizing $W$. A calculation shows that $P^{\prime}$ does not act linearly on $\mathcal{L}(V)_{W}$ already for $k=3$. 
condition $0 \in L$ corresponds to the condition $d F(0)=0$, and the condition $T_{0}(L)=W$ corresponds to vanishing of the second derivatives of $F$ at 0 . This proves the lemma.

We end this section with the definition of a Lagrange singularity type. Consider the subgroup $H$ of $\operatorname{Aut}(V)$ consisting of the holomorphic symplectomorphisms preserving the fibration $\varrho: V \rightarrow W$. Such symplectomorphisms are compositions of maps induced by biholomorphisms of the base and differentials of functions on the base (see [2, §18.5, Theorem, p. 284]).

We say, following $[2, \S 18.6]$, that two jets of Lagrangian submanifolds are Lagrangian equivalent if they belong to the same orbit of $H$.

We study not only individual orbits of the group $H$ but also families of orbits. Moreover, together with an orbit we are forced to consider its closure. Therefore we introduce the following definition.

Definition 3. A Lagrange singularity type $\Sigma$ is any closed pure-dimensional algebraic subset of $\mathcal{L}(V)$ which is invariant with respect to the action of $H$.

(In other words, a Lagrange singularity type is a closed algebraic set which is the union of some Lagrangian equivalence classes.)

For instance, the closure of the orbit of the singularity $A_{2}$ is described by the condition $\operatorname{corank}\left(D \varrho_{\mid L}(0)\right) \geq 1$. Similarly, the closure of the orbit of the singularity $D_{4}$ is given by the condition $\operatorname{corank}\left(D \varrho_{\mid L}(0)\right) \geq 2$. The singularity class $P_{8}$ is not the closure of a single orbit. The family $P_{8}$ has one parameter (i.e. it has modality 1 ). The orbit of a germ belonging to $P_{8}$ has codimension 7 , while the singularity type $P_{8}$ has codimension 6 . It can be described by the condition $\operatorname{corank}\left(D \varrho_{\mid L}(0)\right) \geq 3$. To define the singularity type $A_{3}$ one has to consider the degeneracy locus $S \subset L$ of the differential $D \varrho_{\mid L}$. The singularity type $A_{3}$ consists of the jets of $L$ for which $S$ is singular or $\varrho_{\mid S}$ is not an immersion. These conditions can be translated into algebraic equations in $\mathcal{L}(V)$.

In the literature $([2, \S 21.3])$, one can find the notion of stable Lagrangian equivalence classes. They are classified in small codimensions (loc. cit.).

3. Lagrangian Grassmannians and $\widetilde{Q}$-functions. We start with recollections on $\widetilde{Q}$-functions from [15].

Let $\mathbb{X}$ be an alphabet $\left({ }^{2}\right)$. We shall denote by $\mathbb{X}^{2}$ the alphabet consisting of the squares of elements of $\mathbb{X}$. Given an alphabet $\mathbb{X}$ of variables, we shall denote by $\operatorname{Sym}(\mathbb{X})$ the ring of symmetric functions in $\mathbb{X}$. Given any alphabet

$\left({ }^{2}\right)$ By an alphabet we understand a finite multiset of elements in a commutative ring. 
$\mathbb{X}=\left\{x_{1}, x_{2}, \ldots\right\}$, we set

$$
\widetilde{Q}_{i}(\mathbb{X})=e_{i}(\mathbb{X})=\sum_{j_{1}<\cdots<j_{i}} x_{j_{1}} \cdots x_{j_{i}},
$$

the $i$ th elementary symmetric function in $\mathbb{X}$.

Given two nonnegative integers $i \geq j$, we put

$$
\widetilde{Q}_{i, j}(\mathbb{X})=\widetilde{Q}_{i}(\mathbb{X}) \widetilde{Q}_{j}(\mathbb{X})+2 \sum_{p=1}^{j}(-1)^{p} \widetilde{Q}_{i+p}(\mathbb{X}) \widetilde{Q}_{j-p}(\mathbb{X}) .
$$

For example, we have $\widetilde{Q}_{i, i}(\mathbb{X})=e_{i}\left(\mathbb{X}^{2}\right)$.

Given any partition $I=\left(i_{1} \geq \cdots \geq i_{h} \geq 0\right)$, where we can assume $h$ to be even, we set

$$
\widetilde{Q}_{I}(\mathbb{X})=\operatorname{Pfaffian}(M),
$$

where $M=\left(m_{p, q}\right)$ is the $h \times h$ skew-symmetric matrix with

$$
m_{p, q}=\widetilde{Q}_{i_{p}, i_{q}}(\mathbb{X})
$$

for $1 \leq p<q \leq h$.

For an alphabet $\mathbb{X}=\left\{x_{1}, x_{2}, \ldots\right\}$ of degree 1 variables, the degree of $\widetilde{Q}_{I}(\mathbb{X})$ is equal to $|I|:=i_{1}+\cdots+i_{h}$.

For a fixed positive integer $n$, let $\mathbb{X}_{n}$ be an alphabet of $n$ variables of degree 1 . Then the set $\left\{\widetilde{Q}_{I}\left(\mathbb{X}_{n}\right)\right\}$ indexed by all partitions such that $i_{1} \leq n$ forms an additive basis of the $\operatorname{ring} \operatorname{Sym}\left(\mathbb{X}_{n}\right)$ (see [15]). We shall say that a partition is strict if its parts are distinct. Then the set $\left\{\widetilde{Q}_{I}\left(\mathbb{X}_{n}\right)\right\}$ indexed by all strict partitions such that $i_{1} \leq n$ forms a basis of the $\operatorname{ring} \operatorname{Sym}\left(\mathbb{X}_{n}\right)$ as a free $\operatorname{Sym}\left(\mathbb{X}_{n}^{2}\right)$-module (loc. cit.). The same assertions hold for a countable alphabet of variables without restriction on $i_{1}$.

Let $c_{1}, c_{2}, \ldots$ be a sequence of commuting variables, where $\operatorname{deg}\left(c_{i}\right)=i$, and let $\mathbb{X}=\left\{x_{1}, x_{2}, \ldots\right\}$ be an alphabet of degree 1 variables. We get a ring isomorphism

$$
\Phi: \operatorname{Sym}(\mathbb{X}) \rightarrow \mathbb{Z}\left[c_{1}, c_{2}, \ldots\right]
$$

by setting $\Phi\left(e_{i}(\mathbb{X})\right)=c_{i}$ for $i=1,2, \ldots$.

Given a partition $I$, we put

$$
\widetilde{Q}_{I}=\Phi\left(\widetilde{Q}_{I}(\mathbb{X})\right) .
$$

If $E$ is a vector bundle, then we define

$$
\widetilde{Q}_{I}(E)=\widetilde{Q}_{I}(\mathbb{X}),
$$

where $\mathbb{X}$ is the alphabet of the Chern roots of $E$. In other words, $\widetilde{Q}_{I}(E)$ is equal to $\widetilde{Q}_{I}$, where $c_{i}$ is specialized to $c_{i}(E)$, the $i$ th Chern class of $E$, $i=1,2, \ldots$. 
REMARK 4. The family of $\widetilde{Q}$-functions was invented and investigated in [15] when studying Lagrangian degeneracy loci. It is modelled on the classical Schur Q-functions (see, e.g., [14]). More precisely, for a strict partition $I$, the Schur $Q$-function of a vector bundle $E$ is obtained from $\widetilde{Q}_{I}$ by the substitution

$$
Q_{I}(E)=\widetilde{Q}_{I}\left(E-E^{*}\right) .
$$

See also Remark 5.3 in [12] for another link between these two families of functions. We refer the reader to [15] and [12] for details.

We shall now use $\widetilde{Q}$-functions to describe some cohomological properties of the Lagrangian Grassmannian $L G(V)$. First, we recall a presentation of the cohomology ring of $L G(V)$ by generators and relations that goes back to Borel [4].

Proposition 5. With the above notation, we have

$$
H^{*}(L G(V), \mathbb{Z}) \cong \mathbb{Z}\left[c_{1}, \ldots, c_{n}\right] /\left(\widetilde{Q}_{i, i}\right)_{i=1, \ldots, n} .
$$

(Here the $c_{i}$ 's correspond to the Chern classes of the dual of the tautological subbundle on $L G(V)$.)

The Lagrangian Grassmannian $L G(V)$ has an algebraic cell decomposition which is a particular case of the classical Schubert-Bruhat cell decomposition, but here it admits the following concrete form. Suppose that a general flag

$$
V_{\bullet}: V_{1} \subset \cdots \subset V_{n} \subset V
$$

of isotropic subspaces with $\operatorname{dim} V_{i}=i$ is given (i.e., equivalently, $V_{n}$ is Lagrangian). Given a partition $I=\left(n \geq i_{1}>\cdots>i_{h}>0\right)$, we define

$$
\Omega_{I}\left(V_{\bullet}\right)=\left\{L \in L G(V): \operatorname{dim}\left(L \cap V_{n+1-i_{p}}\right) \geq p, p=1, \ldots, h\right\} .
$$

This is a Schubert variety associated with $I$. Note that

$$
\operatorname{codim}\left(\Omega_{I}\left(V_{\bullet}\right), L G(V)\right)=|I|,
$$

and the cohomology class of $\Omega_{I}\left(V_{\bullet}\right)$, denoted by $\Omega_{I}$, does not depend on the choice of the flag $V_{\bullet}$. Let us recall the following expression for $\Omega_{I}$ in terms of $\widetilde{Q}$-functions.

TheOREm 6 ([14, Sect. 6$])$. Let $V$ be a $2 n$-dimensional linear symplectic space. Then in $H^{2|I|}(L G(V), \mathbb{Z})$, we have

$$
\Omega_{I}=\widetilde{Q}_{I}\left(R^{*}\right),
$$

where $R$ is the tautological subbundle on $L G(V)$.

(In [14, Sect. 6], this result was given in terms of the special Schubert classes $\Omega_{i}$ but $\Omega_{i}=c_{i}\left(R^{*}\right)$.)

Since Schubert varieties are the closures of cells of a cellular decomposition of $L G(V)$, the Schubert classes $\left\{\Omega_{I}\right\}$, with $I$ strict and $i_{1} \leq n$, form a 
$\mathbb{Z}$-basis of $H^{*}(L G(V), \mathbb{Z})$. Hence also the polynomials $\left\{\widetilde{Q}_{I}\left(R^{*}\right)\right\}$ indexed by the partitions from the same set have this property.

In the proof of Theorem 9 below, we shall use the following result. For a strict partition $I=\left(n \geq i_{1}>\cdots>i_{h}>0\right)$, we denote by $I^{\prime}$ the strict partition whose set of parts complements $\left\{i_{1}, \ldots, i_{h}\right\}$ in $\{1, \ldots, n\}$.

Proposition 7. For a strict partition $I$ with $i_{1} \leq n$, there exists a unique strict partition $J$ with $j_{1} \leq n$ and $|J|=\operatorname{dim} L G(V)-|I|$ for which

$$
\widetilde{Q}_{I}\left(R^{*}\right) \cdot \Omega_{J} \neq 0 .
$$

In fact, this $J$ is equal to $I^{\prime}$, and we have

$$
\int_{L G(V)} \widetilde{Q}_{I}\left(R^{*}\right) \cdot \Omega_{I^{\prime}}=1 .
$$

By virtue of Theorem 6 , this proposition follows, e.g., from [15, Theorem 5.23]. See also Example 4.2(5) in [14].

4. Thom polynomials of Lagrange singularities and $\widetilde{Q}$-functions. A Lagrange singularity type $\Sigma \subset \mathcal{L}(V)$ defines the cohomology class

$$
[\Sigma] \in H^{*}(\mathcal{L}(V), \mathbb{Z}) \cong H^{*}(L G(V), \mathbb{Z}) .
$$

Suppose that this class is equal to

$$
\sum_{I} \alpha_{I} \widetilde{Q}_{I}\left(R^{*}\right)
$$

where the sum runs over strict partitions $I$ with $i_{1} \leq n$, and $\alpha_{I} \in \mathbb{Z}$ (it is important here to use the bundle $R^{*}$ ). Then

$$
\mathcal{T}^{\Sigma}:=\sum_{I} \alpha_{I} \widetilde{Q}_{I}
$$

is called the Thom polynomial associated with the Lagrange singularity type $\Sigma$.

EXAmple 8 . We list here the $\widetilde{Q}$-function expansions of the Thom polynomials of some Lagrange singularities. They were computed in [11] in the basis of monomials in Chern classes.

$$
\begin{array}{ll}
A_{2}: & \widetilde{Q}_{1} \\
A_{3}: & 3 \widetilde{Q}_{2} \\
D_{4}: & \widetilde{Q}_{21} \\
D_{6}: & 12 \widetilde{Q}_{32}+24 \widetilde{Q}_{41} \\
A_{7}: & 135 \widetilde{Q}_{321}+1275 \widetilde{Q}_{42}+2004 \widetilde{Q}_{51}+2520 \widetilde{Q}_{6} \\
P_{8}: & \widetilde{Q}_{321} .
\end{array}
$$


Note that all the coefficients in the formulas of Example 8 are nonnegative. For a more extensive list of examples, see Section 5.

The Thom polynomials of the singularities $A_{2}, D_{4}$ and $P_{8}$ are equal to single $\widetilde{Q}$-functions because they are defined by conditions involving only the differential of $\varrho_{\mid L}$, that is, by conditions defining single Schubert varieties in the Lagrangian Grassmannian.

For example, $P_{8}$ is defined by the condition $\operatorname{corank}\left(D \varrho_{\mid L}(0)\right) \geq 3$. This singularity type is the closure of a one-dimensional family of orbits. Therefore the germs of the type $P_{8}$ are not stable in the sense of $[2, \S 21.3]$. On the other hand, $P_{8}$ is worth mentioning since it is the first example of modality that appears in the classification. The Thom polynomial $\widetilde{Q}_{321}$ is an obstruction to deforming the map to one without this singularity.

We now state our main result.

THEOREM 9. For any Lagrange singularity type $\Sigma$, all the coefficients $\alpha_{I}$ in (13) are nonnegative.

For the proof of this theorem, we need several preliminary results.

First of all, we shall use the nonnegativity property of globally generated bundles [7] (see also [6], [13]). Let $E$ be a vector bundle on a variety $X$. By a cone in $E$, we mean a subvariety of $E$ which is stable under the natural $\mathbb{G}_{m}$-action on $E$. If $C \subset E$ is a cone, then one may intersect its cycle $[C]$ with the zero-section of the vector bundle:

$$
z(C, E):=s_{E}^{*}([C])
$$

where $s_{E}^{*}: H^{*}(E, \mathbb{Z}) \rightarrow H^{*}(X, \mathbb{Z})$ is induced by the zero-section $s_{E}: X \rightarrow E$.

We now record the following variant of a result from [7].

Lemma 10. Let $\pi: E \rightarrow X$ be a globally generated bundle on a proper homogeneous variety $X$. Let $C$ be a cone in $E$, and let $Z$ be any algebraic cycle in $X$ of the complementary dimension. Then the intersection $[C] \cdot[Z]$ is nonnegative.

Proof. We decompose $C$ into Whitney strata $C_{i}$, each stratum fibered over $S_{i} \subset X$ (in $C^{\infty}$-topology). Note that $\pi^{-1} Z$ is transverse to $C_{i}$ if and only if $Z$ is transverse to $S_{i}$. (One has to stratify the set $Z$ into Whitney strata and check transversality of each pair of strata. For the notion of transversality of stratified cycles and their intersections, see [9].) Since $X$ is homogeneous, we can move $Z$ to make it transverse to each $S_{i}$. Therefore, we can assume that $\pi^{-1} Z$ is transverse to $C$. Then we have

$$
[C] \cdot[Z]=[C] \cdot\left[\pi^{-1} Z\right] \cdot[X]=\left[C \cap \pi^{-1} Z\right] \cdot[X] .
$$

The last number is equal to the degree of the cone class $z\left(C \cap \pi^{-1} Z, E\right)$, which is nonnegative by $[7$, Theorem $1(\mathrm{~A})]$. 
Our next aim, in the proof of Theorem 9 , is to determine the normal bundle of $L G(V)$ in $\mathcal{L}(V)$. To this end, we need a general result about actions of linear transformations on jets. Let $\mathcal{J}$ be the space of jets (of order $k$ fixed at the beginning) of functions $f:\left(\mathbb{C}^{m}, 0\right) \rightarrow\left(\mathbb{C}^{n}, 0\right)$ satisfying the condition $\operatorname{Df}(0)=0$.

Let $A: \mathbb{C}^{n} \rightarrow \mathbb{C}^{m}$ be a linear map. The map $A$ acts on $\mathbb{C}^{m} \oplus \mathbb{C}^{n}$ by

$$
(q, p) \mapsto(q+A p, p)
$$

and it acts on $\mathcal{J}$ by transforming the graphs of functions. (Note that the action of $A$ is well defined because $D f(0)=0$.) More precisely, the image of the function $f$ is the function $f_{A}$ satisfying the following implicit equation:

$$
f\left(q+A f_{A}(q)\right)=f_{A}(q) .
$$

We state

Proposition 11. The derivative at 0 of $A$ acting on $\mathcal{J}$ is equal to the identity.

(We shall use this proposition in the proof of Lemma 12, where the setting stems from Section 2; in particular, there $m=n$ and $q$ (resp. $p$ ) are the coordinates of $W$ (resp. of $W^{*}$ ) used in that section.)

Proof. We compute the derivative of $A$ in the direction of an arbitrary $f \in \mathcal{J}$. We set

$$
g_{t}=A(t f) \text {. }
$$

By (16), the function $g_{t}$ is given by the implicit equation

$$
t f\left(q+A g_{t}(q)\right)=g_{t}(q) .
$$

We want to show that $g_{t} / t \rightarrow f$ as $t \rightarrow 0$. Since we assume

$$
g_{t}(0)=f(0)=0 \text {, }
$$

it is enough to show that $D g_{t} / t \rightarrow D f$ as $t \rightarrow 0$. Differentiating (18) gives

$$
t D f \circ\left(\mathrm{Id}+A \circ D g_{t}\right)=D g_{t} .
$$

Hence, we get

$$
D g_{t}=(\operatorname{Id}-t D f \circ A)^{-1} \circ t D f .
$$

It follows from (20) that $D g_{t} / t \rightarrow D f$ as $t \rightarrow 0$, and the proposition has been proved.

Let us come back to the setting of Section 2, and put

$$
\mathcal{L}=\mathcal{L}(V) \quad \text { and } \quad G=L G(V) .
$$

Recalling that $R$ denotes the tautological vector bundle on $G$, we give the following description of the normal bundle of $G$ in $\mathcal{L}$. 
LEMma 12. We have a natural isomorphism

$$
N_{G} \mathcal{L} \cong \bigoplus_{i=3}^{k+1} \operatorname{Sym}^{i}\left(R^{*}\right) .
$$

Proof. Let $L \in G$ be a Lagrangian linear subspace. Let us choose a splitting

$$
V \cong L \oplus L^{*}
$$

Using this splitting, by Lemma 2 we construct an isomorphism

$$
\pi^{-1}(L) \cong \bigoplus_{i=3}^{k+1} \operatorname{Sym}^{i}\left(L^{*}\right) .
$$

Two splittings of the exact sequence

$$
0 \rightarrow L \rightarrow V \rightarrow L^{*} \rightarrow 0
$$

differ by a linear map $A: L^{*} \rightarrow L$. By Proposition 11, the action of $A$ on the tangent space to jets is the identity. Hence the isomorphism (23) does not depend on the choice of the splitting (22), and we have (globally) a natural isomorphism (21).

We are now ready to complete the proof of Theorem 9 .

Suppose that $\Sigma$ is a Lagrange singularity class (in fact, it can be any algebraic pure-dimensional cycle in $\mathcal{L}$ ). Let $i: G \hookrightarrow \mathcal{L}$ be the inclusion, and denote by

$$
i^{*}: H^{*}(\mathcal{L}, \mathbb{Z}) \rightarrow H^{*}(G, \mathbb{Z})
$$

the induced map on cohomology rings. We have to examine the coefficients $\alpha_{I}$ of the expression

$$
i^{*}[\Sigma]=\sum \alpha_{I} \widetilde{Q}_{I}\left(R^{*}\right)
$$

Let us fix a strict partition $I$ with $i_{1} \leq n$. By invoking Proposition 7 , the coefficient $\alpha_{I}$ is equal to

$$
i^{*}[\Sigma] \cdot \Omega_{I^{\prime}}
$$

(intersection in $G$ ). Since the cohomology ring and the Chow ring of $G$ are equal, we can compute (25) using the Chow groups. Let

$$
C=C_{G \cap \Sigma} \Sigma \subset N_{G} \mathcal{L}
$$

be the normal cone of $G \cap \Sigma$ in $\Sigma$. Denote by $j: G \hookrightarrow N_{G} \mathcal{L}$ the zero-section inclusion. By deformation to the normal cone $[6, \S 6.1, \S 6.2]$, we have in $A_{*} G$ the equality

$$
i^{*}[\Sigma]=j^{*}[C]
$$


where $i^{*}$ and $j^{*}$ are the pull-back maps of the corresponding Chow groups (see [6]). It follows that

$$
\alpha_{I}=[C] \cdot \Omega_{I^{\prime}}
$$

(intersection in $N_{G} \mathcal{L}$ ). The bundle $R^{*}$ is globally generated; therefore, by Lemma 12 , the vector bundle $N_{G} \mathcal{L}$ is globally generated. The Lagrangian Grassmannian $G=L G(V)$ is a homogeneous space with respect to the action of the symplectic group $S p(V)$. By Lemma 10, applied to the bundle $N_{G} \mathcal{L} \rightarrow G$, the intersection $[C] \cdot \Omega_{I^{\prime}}$ is nonnegative.

This ends the proof of Theorem 9.

REMARK 13. We may compare jets of Lagrangian manifolds by increasing the number of variables. For a Lagrangian submanifold $L \subset V=W \oplus W^{*}$, we consider

$$
L \oplus \mathbb{C} \subset V \oplus\left(\mathbb{C} \oplus \mathbb{C}^{*}\right) .
$$

This induces an embedding of $\mathcal{L}\left(W \oplus W^{*}\right)$ into $\mathcal{L}\left(W^{\prime} \oplus W^{\prime *}\right)$, where $W^{\prime}=$ $W \oplus \mathbb{C}$. In this way, we obtain a chain of inclusions

$$
\mathcal{L}(V)=\mathcal{L}_{0} \subset \mathcal{L}_{1} \subset \cdots \subset \mathcal{L}_{r} \subset \cdots
$$

where $\mathcal{L}_{r}=\mathcal{L}\left(\left(W \oplus \mathbb{C}^{r}\right) \oplus\left(W \oplus \mathbb{C}^{r}\right)^{*}\right)$. We say that the singularity type $\Sigma$ is closed with respect to suspension if one can find a sequence of singularity classes $\Sigma_{r} \subset \mathcal{L}_{r}$ for $r \geq 0$ such that

$$
\Sigma_{r}=\Sigma_{r+1} \cap \mathcal{L}_{r}
$$

and $\Sigma_{0}=\Sigma$. Moreover, we assume that the property (27) holds at the cohomological level, i.e. the restriction $H^{*}\left(\mathcal{L}_{r+1}, \mathbb{Z}\right) \rightarrow H^{*}\left(\mathcal{L}_{r}, \mathbb{Z}\right)$ maps the cohomology class $\left[\Sigma_{r+1}\right]$ to $\left[\Sigma_{r}\right]$. (This holds when $\Sigma_{r+1}$ and $\mathcal{L}_{r}$ intersect transversely along some smooth open and dense subset of $\Sigma_{r}$.)

Suppose now that $E$ is a symplectic manifold, and $E \rightarrow B$ is a fibration with Lagrangian fibers. (Such objects have recently been widely investigated. We do not intend to survey this activity here, but refer the reader to, e.g., [10] and the references therein.) Let, in addition, $L \subset E$ be a Lagrangian submanifold. We denote by $\varrho$ the restriction of the projection to $L$. We study the singular points of the projection $\varrho$. The definition of the singularity type (invariance with respect to change of coordinates) allows us to define the singular points of $\varrho$ of type $\Sigma$. Denote the set of those singular points by $\Sigma(\varrho)$. Assume that the singularity type is closed with respect to suspension (see $(27))$. Then, if the map $\varrho$ satisfies suitable transversality conditions, the class $[\Sigma(\varrho)] \in H^{*}(L, \mathbb{Z})$ is equal to $\mathcal{T}^{\Sigma}$ applied to the virtual bundle $T^{*} L-\varrho^{*} T^{*} B$. See also [11, Theorem 2 and $\left.\S 3.1\right]$.

REMARK 14. In the case of Legendre singularities, using some refinement of our methods, we can get the following positivity result (not as transparent as the one in the Lagrangian case). We adopt the definition of the Thom 
polynomial of a Legendre singularity class from [11, p. 730] $\left(^{3}\right)$. That definition makes use of the classifying space of Legendre singularities (loc. cit.). Let $\xi$ be the canonical line bundle on $B U(1)$, appearing in that definition. Let $\mathbf{1}^{n}$ denote the rank $n$ trivial bundle. The Thom polynomial of a Legendre singularity class can be uniquely represented as a $\mathbb{Z}$-linear combination

$$
\sum_{j \geq 0} \sum_{I} \alpha_{I j} \widetilde{Q}_{I}\left(\left(T^{*} L-\mathbf{1}^{n}\right) \otimes \xi^{1 / 2}\right) \cdot t^{j},
$$

where

$$
t:=\frac{1}{2} c_{1}(\xi) \in H^{2}\left(B U(1), \mathbb{Z}\left[\frac{1}{2}\right]\right),
$$

the second sum is over strict partitions $I$, and $\alpha_{I j} \in \mathbb{Z}$. We then have

$$
\alpha_{I j} \geq 0
$$

for any strict partition $I$ and $j \geq 0$. In other words, we get a positivity result after "perturbing" the argument " $T^{*} L$ " by subtracting a bundle and twisting by some line bundle. If we formally assume that $\xi$ is trivial, then we get the Lagrangian case.

5. Examples. We now list the Thom polynomials of Lagrange and Legendre singularities up to codimension 6 . These polynomials were computed in [11] in the basis of square free monomials in the Chern classes of the bundles involved. We represent them as $\mathbb{Z}$-linear combinations of the products of the "twisted" $\widetilde{Q}$-functions from (28):

$$
\widetilde{Q}_{I}:=\widetilde{Q}_{I}\left(\left(T^{*} L-\mathbf{1}^{n}\right) \otimes \xi^{1 / 2}\right)
$$

(note the difference in notation with respect to (6)), and powers of $t=\frac{1}{2} c_{1}(\xi)$. The bold terms give the Thom polynomials of the corresponding Lagrange singularities.

$$
\begin{array}{ll}
A_{2}: & \widetilde{\mathbf{Q}}_{\mathbf{1}} \\
A_{3}: & \mathbf{3} \widetilde{\mathbf{Q}}_{\mathbf{2}}+t \widetilde{Q}_{1} \\
A_{4}: & \mathbf{3} \widetilde{\mathbf{Q}}_{\mathbf{2 1}}+\mathbf{1 2} \widetilde{\mathbf{Q}}_{\mathbf{3}}+t 10 \widetilde{Q}_{2}+t^{2} 2 \widetilde{Q}_{1} \\
D_{4}: & \widetilde{\mathbf{Q}}_{\mathbf{2 1}} \\
A_{5}: & \mathbf{2 7} \widetilde{\mathbf{Q}}_{\mathbf{3 1}}+\mathbf{6 0 0} \widetilde{\mathbf{Q}}_{\mathbf{4}}+t\left(22 \widetilde{Q}_{21}+86 \widetilde{Q}_{3}\right)+t^{2} 40 \widetilde{Q}_{2}+t^{3} 6 \widetilde{Q}_{1} \\
D_{5}: & \mathbf{6} \widetilde{\mathbf{Q}}_{\mathbf{3 1}}+t 4 \widetilde{Q}_{21} \\
A_{6}: & \mathbf{8 7} \widetilde{\mathbf{Q}}_{\mathbf{3 2}}+\mathbf{2 2 8} \widetilde{\mathbf{Q}}_{\mathbf{4 1}}+\mathbf{3 6 0} \widetilde{\mathbf{Q}}_{\mathbf{5}}+t\left(343 \widetilde{Q}_{31}+756 \widetilde{Q}_{4}\right)+t^{2}\left(151 \widetilde{Q}_{21}+584 \widetilde{Q}_{3}\right)+ \\
& t^{3} 196 \widetilde{Q}_{2}+t^{4} 24 \widetilde{Q}_{1} \\
D_{6}: & \mathbf{1 2} \widetilde{\mathbf{Q}}_{\mathbf{3 2}}+\mathbf{2 4} \widetilde{\mathbf{Q}}_{\mathbf{4 1}}+t 32 \widetilde{Q}_{31}+t^{2} 12 \widetilde{Q}_{21} \\
E_{6}: & \mathbf{9} \widetilde{\mathbf{Q}}_{\mathbf{3 2}}+\mathbf{6} \widetilde{\mathbf{Q}}_{\mathbf{4 1}}+t 9 \widetilde{Q}_{31}+t^{2} 3 \widetilde{Q}_{21}
\end{array}
$$

$\left({ }^{3}\right)$ Our reference for Legendre singularities and characteristic classes is [11, §3.2]. 


$$
\begin{aligned}
A_{7}: & \mathbf{1 3 5} \widetilde{\mathbf{Q}}_{\mathbf{3 2 1}}+\mathbf{1 2 7 5} \widetilde{\mathbf{Q}}_{\mathbf{4 2}}+\mathbf{2 0 0 4} \widetilde{\mathbf{Q}}_{\mathbf{5 1}}+\mathbf{2 5 2 0} \widetilde{\mathbf{Q}}_{\mathbf{6}}+t\left(7052 \widetilde{Q}_{5}+4399 \widetilde{Q}_{41}+\right. \\
& \left.1693 \widetilde{Q}_{32}\right)+t^{2}\left(3445 \widetilde{Q}_{31}+7748 \widetilde{Q}_{4}\right)+t^{3}\left(1046 \widetilde{Q}_{21}+4172 \widetilde{Q}_{3}\right)+t^{4} 6388 \widetilde{Q}_{2}+ \\
& t^{5} 5400 \widetilde{Q}_{1} \\
D_{7}: & \mathbf{2 4} \widetilde{\mathbf{Q}}_{\mathbf{3 2 1}}+\mathbf{1 2 0} \widetilde{\mathbf{Q}}_{\mathbf{4 2}}+\mathbf{1 4 4} \widetilde{\mathbf{Q}}_{\mathbf{5 1}}+t\left(152 \widetilde{Q}_{32}+288 \widetilde{Q}_{41}\right)+t^{2} 208 \widetilde{Q}_{31}+t^{3} 56 \widetilde{Q}_{21} \\
E_{7}: & \mathbf{9} \widetilde{\mathbf{Q}}_{\mathbf{3 2 1}}+\mathbf{6 0} \widetilde{\mathbf{Q}}_{\mathbf{4 2}}+\mathbf{2 4} \widetilde{\mathbf{Q}}_{\mathbf{5 1}}+t\left(56 \widetilde{Q}_{41}+66 \widetilde{Q}_{32}\right)+t^{2} 42 \widetilde{Q}_{31}+t^{3} 10 \widetilde{Q}_{21} \\
P_{8}: & \widetilde{\mathbf{Q}}_{\mathbf{3 2 1}} .
\end{aligned}
$$

Acknowledgements. We thank Özer Öztürk for pointing out some corrections in an earlier version of the paper. We are also grateful to the referee for a careful reading of the manuscript and suggestion of several improvements.

\section{References}

[1] V. I. Arnold, On a characteristic class entering into conditions of quantization, Funktsional. Anal. i Prilozhen. 1 (1967), no. 1, 1-14 (in Russian).

[2] V. I. Arnold, S. M. Gusein-Zade and A. N. Varchenko, Singularities of Differentiable Maps, Vol. I, Monogr. Math. 82, Birkhäuser, 1985.

[3] M. Audin, Classes caractéristiques lagrangiennes, in: Algebraic Topology, Barcelona 1986, Lecture Notes in Math. 1298, Springer, 1987, 1-16.

[4] A. Borel, Sur la cohomologie des espaces fibrés principaux et des espaces homogènes de groupes de Lie compacts, Ann. of Math. 57 (1953), 115-207.

[5] D. B. Fuks, The Maslov-Arnold characteristic classes, Dokl. Akad. Nauk SSSR 178 (1968), 303-306 (in Russian).

[6] W. Fulton, Intersection Theory, Springer, 1984.

[7] W. Fulton and R. Lazarsfeld, Positivity and excess intersections, in: Enumerative and Classical Geometry (Nice, 1981), Progr. Math. 24, Birkhäuser, 1982, 97-105.

[8] —, -, The numerical positivity of ample vector bundles, Ann. of Math. 118 (1983), $35-60$.

[9] M. R. Goresky, Whitney stratified chains and cochains, Trans. Amer. Math. Soc. 267 (1981), 175-196.

[10] J.-M. Hwang, Base manifolds for fibrations of projective irreducible symplectic manifolds, arXiv:0711.3224.

[11] M. Kazarian, Thom polynomials for Lagrange, Legendre, and critical point function singularities, Proc. London Math. Soc. (3) 86 (2003), 707-734.

[12] A. Lascoux and P. Pragacz, Operator calculus for $\widetilde{Q}$-polynomials and Schubert polynomials, Adv. Math. 140 (1998), 1-43.

[13] R. Lazarsfeld, Positivity in Algebraic Geometry, Springer, 2004.

[14] P. Pragacz, Algebro-geometric applications of Schur S- and Q-polynomials, in: Topics in Invariant Theory, Séminaire d'Algèbre Dubreil-Malliavin 1989-1990 (M.-P. Malliavin, ed.), Lecture Notes in Math. 1478, Springer, 1991, 130-191.

[15] P. Pragacz and J. Ratajski, Formulas for Lagrangian and orthogonal degeneracy loci; $\widetilde{Q}$-polynomial approach, Compos. Math. 107 (1997), 11-87.

[16] P. Pragacz and A. Weber, Positivity of Schur function expansions of Thom polynomials, Fund. Math. 195 (2007), 85-95. 
[17] V. A. Vasil'ev, Characteristic classes of Lagrange and Legendre manifolds that are dual to singularities of caustics and wave fronts, Funktsional. Anal. i Prilozhen. 15 (1981), no. 3, 10-22 (in Russian).

Warsaw University of Technology

Pl. Politechniki 1

00-661 Warszawa, Poland

E-mail: emmikosz@mech.pw.edu.pl

Department of Mathematics

Warsaw University

Banacha 2

02-097 Warszawa, Poland

E-mail: aweber@mimuw.edu.pl
Institute of Mathematics Polish Academy of Sciences

Sniadeckich 8 00-956 Warszawa, Poland E-mail: P.Pragacz@impan.pl

Received 23 April 2008;

in revised form 9 August 2008 